\title{
Towards a sustainable aerogel airship: A primer
}

\author{
Nadiir Bheekhun ${ }^{1 *}$, Abd Rahim Abu Talib ${ }^{2}$, Fortunato Cardenas ${ }^{3}$ \\ ${ }^{1}$ Aerospace and Communication Technology Research Group, Department of Engineering and Technology, Faculty of Information Sci- \\ ences and Engineering, Management \& Science University, Shah Alam, Selangor Darul Ehsan, Malaysia \\ ${ }^{2}$ Aerodynamics, Heat Transfer and Propulsion Research Group, Department of Aerospace Engineering, Faculty of Engineering, \\ Universiti Putra Malaysia, 43400 Serdang, Selangor Darul Ehsan, Malaysia \\ ${ }^{3}$ Huvr Trek Group, Green Earth Aerogel Technologies, JosepPla, 2 Torres Diagonal LitoralEdf. B3/1, 08019 Barcelona, Spain \\ *Corresponding author E-mail: nadiir@msu.edu.my
}

\begin{abstract}
Airships are the first craft that realized mankind's dream of controlled, powered flight but have been a forsaken method of air transportation since the invention of heavy-than-air aircraft. However, environmental concerns have urged humanity to think once again over the possibility of deploying these lighter-than-air ships. This paper describes an on-going project thereby a nature-inspired airship, namely Huvr Trek is being designed to address current airborne technological, economical and ecological gaps. It uses the world's best thermal insulator and second lightest material, silica aerogel for inhibiting heat transfer within the balloon for efficient lift as well as in a prototype liquid-fuelled ramjet for propulsion. The airship uses carbon aerogels also as super capacitors for energy storage. Proposed applications of the aerogel airship are and implementations into advertising and tourism, surveillance, environmental monitoring, planetary exploration, cargo transportation, stratospheric observation, medical equipment carrier and telecommunication relay.
\end{abstract}

Keywords: aerogels; airship; energy; insulation; zero emission.

\section{Introduction}

Increasing concerns on volatile prices and potential scarcity of the fossil fuels, coupled with on-going global climate change, have further pronouncing the need for green technology innovations in the aerospace arena. Concepts of renewable energy, super insulation and electric aero-propulsion systems are continuously being brought forward by both academia and industry in order to realise a sustainable low carbon society. History has proven that more efforts have been made towards the heavy-than-air (HTA) craft in comparison to lighter-than-air (LTA) vehicles that are traditionally known as airships or dirigibles. The former category uses fixedwing (aircraft) and rotary-wing (helicopter) to generate the aerodynamic lift by the motion of an aerofoil through the air whilst the latter stays aloft using light gas to create a buoyancy effect, which may be referred to as an aerostatic lift. This distinctive characteristic have allowed them to have longer endurance, higher payloadto-weight ratio with lower fuel consumption.

Airships have witnessed both remarkable triumphs and shocking tragedies. Dirigibles were first materialised in 18th century with the very first recorded flight of a non-rigid dirigible by Jean-Pierre Blanchard it 1784. The word "dirigible" in fact comes from the French word 'diriger' that means "to direct or to steer". The ellipsoidal-shaped balloon consisted of with a rudder, an elevator, and three large airscrews to convert the hand power into propulsion. In 1852 , the first engine-propelled airship, powered by steam was invented by Henri Giffard thereby he flew 27 kilometres. The airship had a length of 143 feet and a diameter of 40 feet. It successfully completed a flight of 17 miles at a speed of $5 \mathrm{mph}$ using a three horsepower engine. Attempts in deriving more powerful and bigger airships continued and in 1900, the golden age of air- ships instigated with the advent the German-made dirigible Zeppelin LZ-1 (Luftschiff Zeppelin). In 1909, the first airliner, DELAG, which stands for DeutsheLuftschiffahrts-Aktiengesellschaft (German Airship Transportation Corporation Ltd), was founded. During 1920s and 1930s, after the World War I, the United States and Britain constructed several airships, almost replicating the original Zeppelins' design. They served as commercial carrier, cargo as well as for military purposes. The era of airships literally ended due to several airship accidents, including the 1937 burning of the Hindenburg, the largest aircraft ever built. It burst into flames and crashed to the ground at Lakehurst, N. J., killing 22 crewmen, 13 passengers and a ground worker. Meanwhile, the first powered heavier-than-air aircraft was already invented by the Wright brothers and significant progresses were being made on fixedwing aircraft showing their efficacy [1].

However, during the past few years, interests have been diverted towards these LTA vehicles while being in quest for alternative solutions for fossil fuelled vehicles and greener air transportation. Advancement of modern techniques, such as composite materials, optimal design, computational fluid dynamics, thermal modelling, vectored thrust, and automatic control, have all brought a resurgence of airships. In terms of applications, both commercial and scientific suggestions for modern airships have been proposed; amongst are in advertising and tourism [2], surveillance [3], environmental monitoring, planetary exploration, heavy-lift cargo transport, stratospheric observation and telecommunication relay. In order to realise these applications of airships, dynamic modelling is essential in order to analyse their flight behaviour and design control systems while optimising their flight trajectories. It is worthy to note that the dynamic modelling of HTA and LTA are dissimilar for a number of reasons including a difference in the lift generation mechanisms, a change in assumption regarding solid- 
fluid interactions such as buoyancy and those related with inertia of the surrounding air and the geometry/structure. Li et al. [4] addressed a state-of-the-art literature review focusing on airship dynamics. The article provided a comprehensive description of the main problems in airship dynamics modelling and summarized the theoretical (analytical or numerical) and semi-empirical techniques to solve these problems. They discussed the implications of the collar triangle in airships technology, in other words, the interaction of aerodynamics/aerostatics, flight dynamics and structural flexibility. In addition, two factors were added into the triangle: atmospheric turbulence and effects of ballonets, as shown in Figure 1 . The thicknesses of the lines and circles quantify the amount of existing research in each discipline for airships.

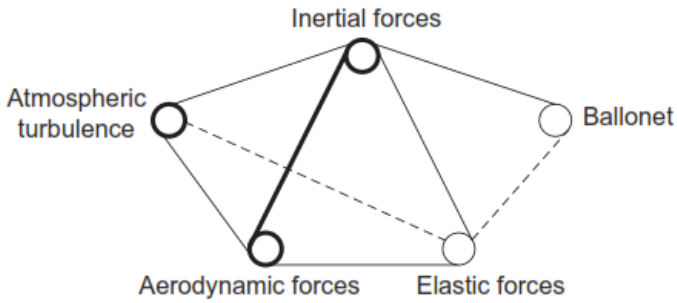

Fig. 1: Interaction of five disciplines in airships technology [4]

On the other hand, aerogels [5] are a class of materials, often considered as a state of matter on their own which are prepared via a sol-gel process followed by drying. An aerogel is essentially a gel in which the liquid phase has been replaced by a gas, usually carbon dioxide, without collapsing the already existing nanoporous structure, thereby avoiding the subsequent shrinkage and cracking of the dried gel. Initially, at the time of invention by Samuel Kistler in 1931, supercritical drying was used to achieve so [6]. Nowadays, ambient and freeze drying technology have been successfully applied as well $[7,8]$. Amongst the various types of aerogels, silica aerogel has the lowest thermal conductivity which dignifies it as the most superior thermal insulator [9], graphene aerogel is the lightest of all materials [10] while carbon aerogel patented by Richard W. Pekala and his colleagues at Lawrence Livermore National Laboratory in 1988 [11], has been appreciated as a very promising material for many energy related applications including hydrogen and electrical storage due to its unique combination of characteristics of high surface area, rich porosity, highly electrically conductive, environmental compatible and chemically stable [12].

These fascinating nanomaterials, which have been known for many decades, are now being tailored accordingly in respective industries to address numerous technological gaps which could not be done decades before due to their relatively high manufacturing cost. Green Earth Aerogels Technologies is one of the leading aerogels manufacturers and uses an agricultural waste product, rice husk ash to synthesis its silica aerogel under ambient conditions. As a result, the cost is cut by almost $80 \%$ compared to synthetically-TEOS manufactured silica aerogels. A benchmarking study was carried out by Bheekhun et al. [13], which demonstrated that GEA-aerogels exhibit comparable nanostructural characteristics with remarkably low thermal conductivity of order 0.015 $\mathrm{W} / \mathrm{mK}$ at $25^{\circ} \mathrm{C}$. Carbon aerogels, on the other hand, are synthesised from rice husk via sol-gel polymerization of resorcinol and formaldehyde, followed by drying and subsequent pyrolysis at an elevated temperature in an inert atmosphere. The resorcinolformaldehyde aerogels (RF) are produced in a way similar to silica aerogels.

The applications of aerogels in aeronautics have been discussed elsewhere [13] and trends have shown that they have potential to be implemented in aero-engines for both thermal and fire insulations. Recently, a multi-layer concept was proposed by Jin et al [14], thereby yttria stabilised zirconia (YSZ) and silica aerogel were used in conjunction to improve the thermal insulation capability of an aerospace graded titanium alloy (Ti-6Al-4V or TC4).
The aerogel was adhered onto the surface of the alloy using organic glue as a transitional layer or bond coat between the substrate TC4 and the upcoming plasma sprayed YSZ. In addition, a single layered aerogel-based plasma sprayed coating was achieved by the authors in a previous work yet to be reported, which demonstrated the possibility to attain a micro-thick layer which consisted of a bimodal microstructure. In other words, the aerogel network had been retained thereby increasing the possibility of the aerogelbased coat to exhibit characteristics almost similar to powdered silica aerogel.

While progress is being made in applying aerogels in HTA vehicles, until now no investigation has been brought forward in considering their applications in LTA crafts. Furthermore, as per the authors' knowledge, aerogels have not yet been applied in airships. The ultralight weight of aerogels, low thermal conductivity and highly electrically conductive of silica and carbon aerogels respectively is taken into account for specific applications. In this context, this paper previews an on-going project thereby ecologically-derived aerogels are used in the construction of a zero emission airship.

\section{Airship specifics}

\subsection{Aerogels}

Rice rusk ash-derived GEAT® 0.125 silica aerogel and GEAT® carbon aerogel have been manufactured at Green Earth Aerogel Technologies, Spain.

\subsection{Design and framework}

The design of the airship is a nature-inspired solution, one from a mussel and horseshoe crab which are well-known for their stability in high winds. The rigid airship, that is, one which has a rigid framework covered by an outer skin or envelope has trusses made with coated and laminated carbon fibre. It extends as long as 32 metres and has width and height of 8 metres.

The airship has a gondola attached to it and contains a number of lead shot bags which are constantly adjusted based on the crew's analysis. The gondola is attached to the envelope sides. Inside the gondola, there a series of controls: the overhead control panel containing controls for communications, fuel, and electrical systems; throttles to regulate engine speed; fuel mixture and heat controls to regulate the degree to which fuel is mixed with air in engine; temperature controls to prevent icing; envelope pressure controls to regulate helium and ballonet air pressure; communication equipment; main instrument panel; navigational instruments; and colour weather radar. The gondola is made up of carbon fibre and glass fibres laminated in vitrified polymer to be fireproof and impermeable and coated metal foil with silica aerogel powder. All in all, these are illustrated in Figure 2.

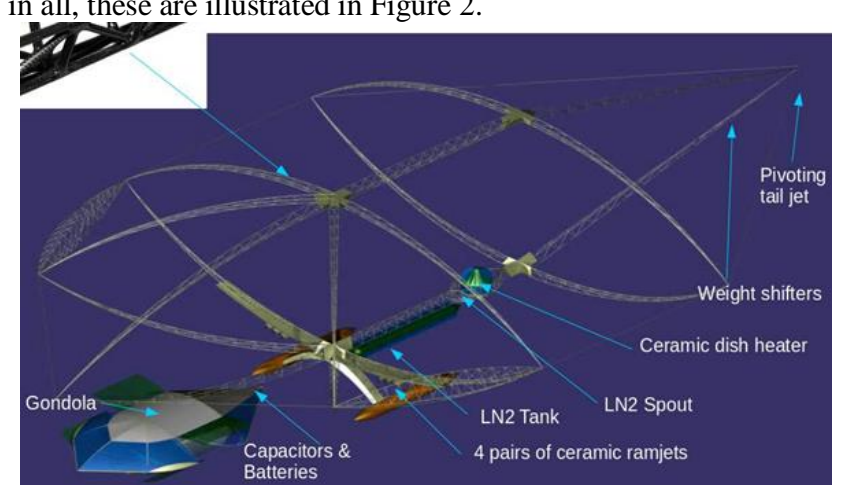

Fig. 2: Huvr Trek aerogel airship framework. 


\subsection{Motion control}

Pitch and roll motion are controlled by two hydraulic springs used to move the weight shifter, which changes the equilibrium of the airship. This is operated from the gondola. Yaw motion is controlled by the rear-end trail thruster (ramjet), which also works using a hydraulic spring, as shown in Figure 3.

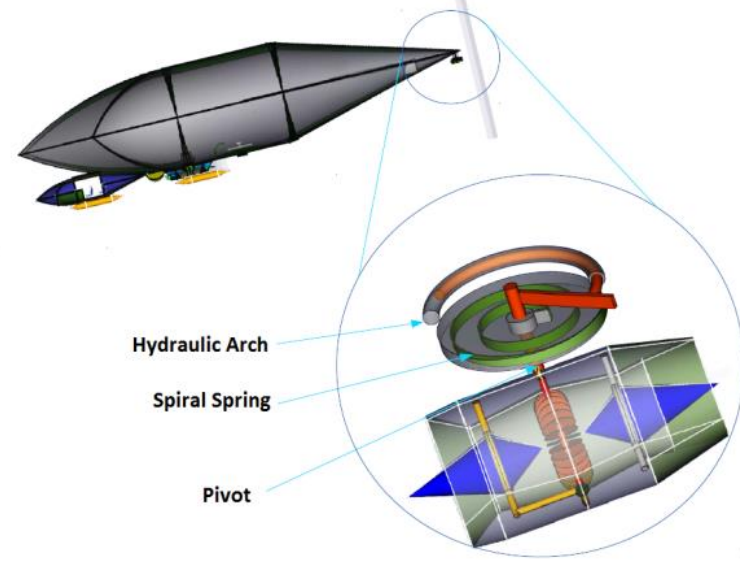

Fig. 3: Pivoting tail ramjet

\subsection{Charging and heating systems}

Unlike most airships, the Huvr Trek airship uses liquid nitrogen as fuel. Its energy density is less than gasoline but more economical and ecological. Power is stored in liquid nitrogen and the fuel tank where liquid nitrogen is stored is equipped with heat exchangers that allow ambient heat to be absorbed by the fuel tank itself. The ambient heat is transferred to the liquid nitrogen which causes the liquid nitrogen to rapidly boil into gaseous nitrogen. As the gaseous nitrogen expands, the gas is projected towards a tesla turbine which converts gas expansion into mechanical energy. At this stage mechanical energy from the turbine is converted again into electrical energy via an electric generator which is mounted to the turbine. The cold nitrogen gas then enters a chamber which leads to the nozzles inside the jet engines. These nozzles are designed to direct cold nitrogen gas toward a ceramic heat exchanger while causing a venturi effect on the front side of the jet engine. The venturi effect together with the rapid thermal expansion of gases inside the chamber of the jet engine produce a combined push and pull effect on the jet engine. The front side of the jet engine will be producing a pulling force caused by the vacuum with the venturi effect and a pushing force is produce by the rapid expanding gases at the rear end of the jet engine. The electricity produced by the electric generator from the tesla turbine is stored in the primary battery storage of the Huvr Trek. This battery is a lithium ion battery which feed a special circuit of carbon aerogel capacitors to multiply the voltage of the electrical charge. The ultra-high voltage of the electrical charge released by the capacitors is then fed to the ceramic magnetron which converts it into plasma energy. The plasma energy is used to heat up ceramic heat exchangers which are used to heat up the cold nitrogen gas in the jet engines and also in the balloon chamber of the airship. Each heat exchanger has its own electrical circuit with ultra-high voltage for its plasma heaters.

As the primary battery of the Huvr Trek is made of lithium ion battery, it can be charged both by the tesla turbine from nitrogen gas expansion and also by direct charging from an electrical grid. To enhance the overall performance of the airship, it is preferred to use direct charging from an electrical source to charge the primary battery and only later use the nitrogen gas during flight. In this manner the life of the battery may be extended without depending too much on nitrogen fuel. When the primary battery is fully charged, it can release energy to the carbon aerogel capacitors and produce plasma energy in the heat exchangers which heats up the nitrogen gas inside the silica aerogel insulated balloon chamber, as illustrated in Figure 4. This charging process contin- ues until the balloon is hot and lift is produced. When the balloon begins to float in the air, it is then that the nitrogen gas is released to the tesla turbine to activate the ceramic jet engines.

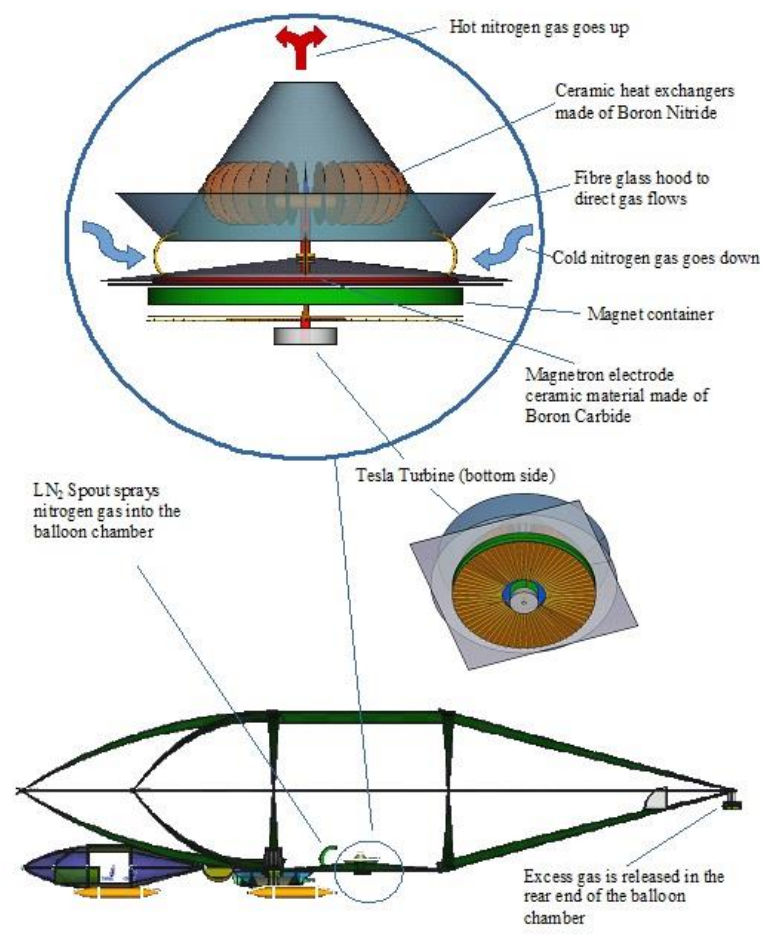

Fig. 4: Plasma balloon heater components.

The CFD analysis is still on-going but nevertheless a primary heat transfer distribution is shown in Figure 5, with temperature ranging from approximately $18^{\circ} \mathrm{C}$ to $32^{\circ} \mathrm{C}$, that is, room temperature after ten minutes of heating. The landing of the airship is accomplished by injecting cold nitrogen inside the balloon. Hot gases are released and replaced by cold nitrogen gas which is denser and heavier to allow a smooth landing either on land, water or snow or ice. The excess gas is released at the tail end of the airship whilst critical volume and pressure are maintained to prevent collapsing of the rigid structure.
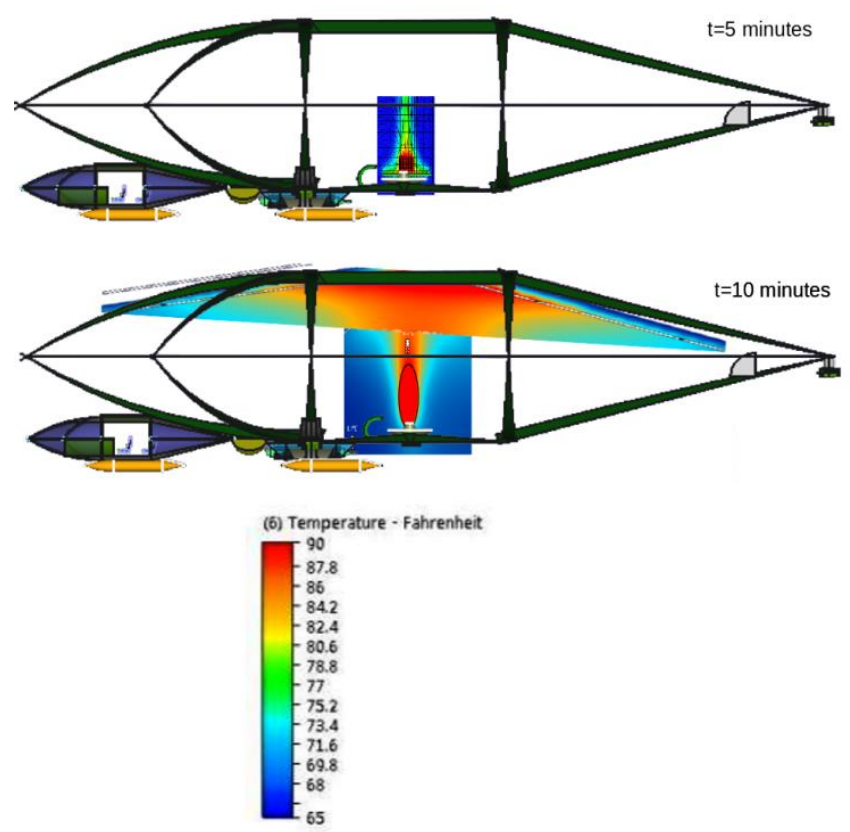

Fig. 5: Simulated heat transfer distribution 


\subsection{Ceramic ramjets}

The ramjets contribute to the propulsion of the airship itself. A magnetron known as boomerang electron accelerated (B.E.A) Magnetron, patented by GEAT, is located just beneath the balloon chamber. Its primary function is to produce plasma energy by accelerating electrons to bombard a ceramic material, which is boron nitride. Consequently all the energy is transformed into heat energy which is then transferred to the heat exchangers. Each ceramic jet engine is also equipped with its own ceramic magnetron. The magnetron in the jet engines is located at the rear end of the nose tip of the jet engines just beneath the nozzles of the nitrogen gas. The function of the magnetron in the jet engine is similar to its function the balloon chamber which is, producing plasma energy and heat up the ceramic heat exchangers. The ceramic magnetron has ceramic electrodes (anode and cathode) which get the polarity of the electrical charge. In between the electrodes are magnetic rings that have increasing magnetic fields which directs the flow of electrons in a spiral shape with decreasing radius towards the centre. It is designed to maximize the path of the election in a curvature to allow it to gain acceleration before colliding to the ceramic material thereby optimizing the kinetic energy of the electron upon collision. The magnetron heater, which is heated prior to the initial start of the ramjet, is powered by aerogel super capacitors as voltage multipliers. Silica aerogel is used to insulate the chamber walls of the jet engine to avoid heat loss and concentrate the energy to gas expansion inside the chamber. In addition, it is also used to insulate the tubes and pipes where the liquid nitrogen and gaseous nitrogen flows within the tank and the ramjet. The principle operation of aerogel-based ceramic ramjet is depicted in Figure 6 while the proposed performance of the airship is tabulated in Table 1.

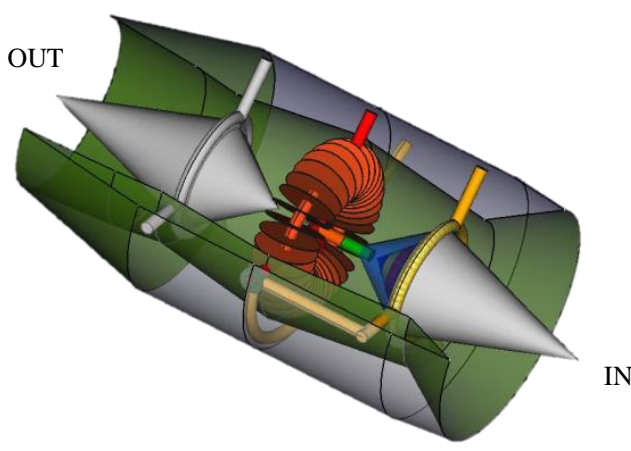

The ceramic magnetron is the hea source of the orange discs. Once the liquid nitrogen gets in contact on the discs, $\mathrm{N}_{2}$ gas rapidly expands towards the rear of the ramiet.

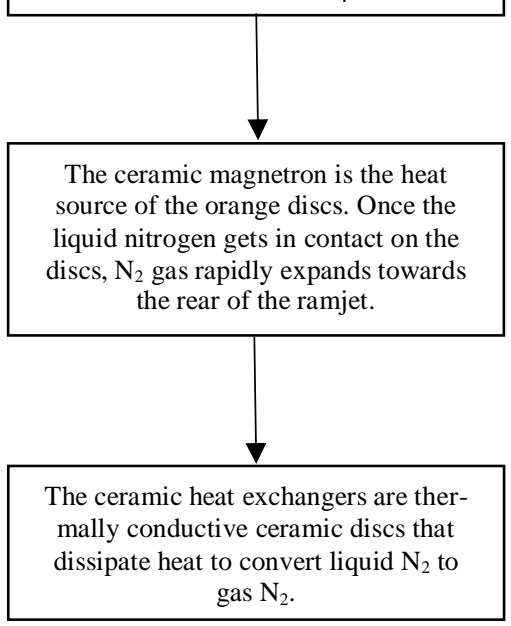

Fig. 6: Principle of operation of aerogel-based ceramic ramjet
Table 1: Intended performance of aerogel airship

\begin{tabular}{|l|l|}
\hline Engine Model & GEAT NitroJet-1A \\
\hline Engine Thrust & $8391 \mathrm{~kg}(18,500 \mathrm{lbs})$ \\
\hline Cruising Speed & $250 \mathrm{~km} / \mathrm{h}$ \\
\hline Maximum Altitude & $1000 \mathrm{~m}$ \\
\hline Fuel Capacity & Yet to be determined \\
\hline Fuel Consumption/hour & Yet to be determined \\
\hline Maximum Range & $1000 \mathrm{~km}$ \\
\hline Engine Thrust & $8391 \mathrm{~kg}(18,500 \mathrm{lbs})$ \\
\hline
\end{tabular}

\section{Conclusion}

Environmental sustainability is nowadays a pronounced driver of innovation. Airships are believed to be a convenient way to address certain challenges in air transportation. They do not require any powerful propulsion systems to stay aloft since all the necessary lift is acquired from the buoyancy of lifting gases. This significantly reduces the power requirements and fuel consumption and thus reduces the overall operating costs. To enhance the performance airship technology, new concepts have been brought forward in this project. Firstly, the airship shape has been adopted from a mussel and horseshoe crab which are well-known for their stability in high winds. Secondly, silica aerogels are used to insulate the trusses made with coated and laminated carbon fibre and the balloon envelope to ensure minimum heat transfer to the atmosphere. Thirdly, liquid nitrogen is opted as the environmental friendly fuel and a silica aerogel-based magnetron is used to generate plasma to be converted into electrical energy which is in turn stored in ecological supercapacitors made up of carbon aerogels. Fourthly, silica aerogel-insulated ramjets are used for propulsion as well as to yaw the airship. The superinsulation of the chamber walls of the jet engine prevents heat loss and concentrates the energy to gas expansion inside the chamber. The tubes and pipes where the liquid nitrogen and gaseous nitrogen flows within the tank and the ramjet are also insulated.

\section{Acknowledgement}

This work is headed by the Huvr Trek Group under Green Earth Aerogel Technologies. The authors would like to thank the Ministry of Higher Education of Malaysia for their financial support to this research study through Fundamental Research Grant Scheme FRGS/TK05/UPM/02/7 No. 5524896.

\section{References}

[1] Liao L \& Pasternak I (2009), A review of airship structural research and development. Progress in Aerospace Sciences 45, 83-96

[2] Oh S, Kang S, Lee K, Ahn S \& Kim E (2006), Flying display: Autonomous blimp with real-time visual tracking and image projection. IEEE/RSJ International Conference on Intelligent Robots and Systems

[3] Colozza A \& Dolce JL (2005), High-altitude, long-endurance airships for coastal surveillance. NASA Technical Report, NASA / TM-2005-213427

[4] Li Y, Nahon M \& Sharf I (2011), Airship dynamics modeling: A literature review. Progress in Aerospace Sciences 47, 217-239

[5] Kistler SS (1931), Coherent expanded aerogels and jellies. Nature 127,741

[6] Kistler SS (1932), Coherent expanded aerogels. Journal of Physical Chemistry 36, 52-64

[7] Bhagat SD, Kim Y, Moon M, Ahn Y \& Yeo J (2007), A costeffective and fast synthesis of nanoporous $\mathrm{SiO}_{2}$ aerogel powders using water-glass via ambient pressure drying route. Solid State Sciences 9, 628-635

[8] Fen SL, Lei M, Sakae T \& Gang X (2012), Low-cost and fast synthesis of nanoporous silica cryogels for thermal insulation applications. Science and Technology of Advanced Materials 13, 035003

[9] Cuce E, Cuce PM, Wood CJ \& Riffat SB (2014), Toward aerogel based thermal superinsulation in buildings: a comprehensive review. Renewable and Sustainable Energy Reviews 34, 273-299 
[10] Worsley MA, Pham TT, Yan A, Shin SJ, Lee JR, Bagge-Hansen M, Mickelson W \& Zettl A (2014), Synthesis and characterization of highly crystalline graphene aerogels. ACS Nano 8, 11013-11022

[11] Pekala RW (1989), Low density, resorcinol-formaldehyde aerogels. Google Patents

[12] Pekala RW, Mayer ST, Kaschmitter JL \& Kong FM (1993), Carbon aerogels: An update on structure, properties and applications. in Sol-Gel Processing and Applications. Springer

[13] Bheekhun N, Abu Talib AR \& Hassan MR (2013), Aerogels in aerospace: An overview. Advances in Materials Science and Engineering 2013, 18

[14] Jin L, Li P, Zhou H, Zhang W, Zhou G \& Wang C (2015), Improving thermal insulation of TC4 using YSZ-based coating and $\mathrm{SiO}_{2}$ aerogel. Progress in Natural Science: Materials International 25, $141-146$ 